\title{
Reacquisition following extinction in appetitive conditioning
}

\author{
SEAN T. RICKER and MARK E. BOUTON \\ University of Vermont, Burlington, Vermont
}

\begin{abstract}
In four experiments utilizing an appetitive conditioning preparation, reacquisition of conditioned responding was found to occur both rapidly and slowly following extinction. In Experiment 1, acquisition of responding to a tone that had been conditioned and extinguished occurred more rapidly than acquisition in either a group that received equivalent exposure to the food unconditioned stimulus or a "rest" control group that received only exposure to the apparatus in the first two phases. However, reacquisition was impaired relative to acquisition in a "learning-experienced" group that had previously received conditioning and extinction with a different stimulus. Experiments 2 and 3 produced similar results, but also found that high responding during reacquisition was confined to trials that followed reinforced, rather than nonreinforced, trials. Experiment 4, in which very few initial conditioning trials were used, produced reacquisition that was slow compared with both learning-experienced and rest controls. The results are consistent with a role for sequential learning: Reacquisition is rapid when animals have learned that reinforced trials signal other reinforced trials.
\end{abstract}

It is often assumed that reacquisition of conditioned responding following extinction occurs more rapidly than acquisition with a novel conditioned stimulus (CS; e.g., McAllister \& McAllister, 1994). The assumption is based, in large part, upon a number of early studies (Brogden, Lipman, \& Culler, 1938; Finch \& Culler, 1935; Frey \& Butler, 1977; Frey \& Ross, 1968; Hilgard \& Marquis, 1935; Hoehler, Kirschenbaum, \& Leonard, 1973; Konorski \& Szwejkowska, 1950, 1952a, 1952b; Smith \& Gormezano, 1965; Szwejkowska, 1950). In these experiments, responding during reconditioning of the CS was compared with responding during the original conditioning phase in the same animals. Rapid reappearance of the conditioned response after extinction was taken as evidence that the CS retains some excitatory strength following extinction, and that this strength transfers positively to the reacquisition phase. The CS was seen as starting with a greater associative strength during reacquisition than during conditioning.

The idea that the CS survives extinction with some excitation is clearly consistent with a variety of evidence (e.g., Bouton, 1991, 1993). However, as Bouton (1986) pointed out, numerous difficulties exist in interpreting the literature on reacquisition. The high level of responding obtained during reacquisition in studies prior to 1986 often could have been due merely to spontaneous recovery. That is, in many early experiments, reacquisition began after a time interval that would have permitted

This research was supported by Grant IBN-9209454 from the National Science Foundation. Experiments 1 and 2 were part of a thesis submitted by the first author in partial fulfillment of the requirements of the master's degree at the University of Vermont. Correspondence should be directed to M. E. Bouton, Department of Psychology, University of Vermont, Burlington, VT 05405. spontaneous recovery to be present at the outset. It is also possible that reinstatement, where unconditioned stimulus (US) presentations in the same context as extinction restore responding to an extinguished CS (Bouton \& Bolles, 1979b; Bouton \& Peck, 1989; Rescorla \& Heth, 1975), or even renewal, where testing an extinguished CS in a different context than that used in extinction results in increased responding (Bouton \& Bolles, 1979a; Bouton \& Ricker, 1994), could have been responsible for the high level of responding observed. Early studies of reacquisition often used massed conditioning and reconditioning trials. With trials spaced closely together, it is possible that stimulus aftereffects of the US could have provided a context for renewal of conditioned responding (Bouton, Rosengard, Achenbach, Peck, \& Brooks, 1993). Early studies had not controlled for any of these effects (for argument, see Bouton, 1986).

In contrast, clear evidence of slow reacquisition has been obtained in the conditioned emotional response (CER) procedure (Bouton, 1986; Bouton \& Swartzentruber, 1989). In these experiments, attempts were made to minimize recovery effects that might complicate interpretation of reconditioning. The general procedure involved giving one group conditioning, extinction, and reconditioning phases with one $\mathrm{CS}$, and comparing the rate of acquisition in the final phase with that of a control group that received conditioning with a novel CS at the same time. (These were the first experiments to compare acquisition in an extinguished CS with acquisition of a novel CS paired with the US at a similar point in the experiment.) Bouton (1986) used a "rest" control, which received exposure to the apparatus but no Pavlovian events during the first two phases. Bouton and Swartzentruber (1989) used a "learning-experienced" control, which received conditioning and extinction of a different CS dur- 
ing the first two phases. The basic finding was that, given enough extinction trials, conditioning in the third phase proceeded more slowly for the group that received conditioning with the extinguished CS than for groups that received a novel CS. Bouton interpreted these findings as indicating that, following extinction, a memory of that phase exists which interferes with subsequent conditioning.

Slow, rather than fast, reacquisition has also been obtained in taste-aversion learning. For example, Danguir and Nicolaidis (1977) found extremely slow reacquisition; their results suggested that an aversion to saccharin could not be relearned following extinction. In contrast, Revusky and Coombes (1979) found that reacquisition did occur. It was rapid compared to acquisition in a rest control group but comparable in rate to acquisition in a learning-experienced control group (Revusky \& Coombes, 1979, Experiment 1). More recent studies (Hart, Bourne, \& Schachtman, 1995) have found reacquisition to occur more slowly than acquisition in a learning-experienced group.

The results obtained with CER and taste-aversion learning contrast sharply with data recently reported in the rabbit nictitating membrane response (NMR) paradigm (Napier, Macrae, \& Kehoe, 1992). In a series of four experiments, Napier et al. addressed the criticisms offered by Bouton (1986) concerning previous research with this method. However, even after controlling for reinstatement, renewal, and spontaneous recovery, they consistently found reacquisition to occur rapidly when compared with a rest control. Rapid reacquisition occurred whether extinction consisted of CS-alone presentations or explicitly unpaired CS and US presentations (ruling out renewal by US presentations as a mechanism in the final phase). Rapid reacquisition also occurred when the CS was subjected to procedures that could have given it an inhibitory value in the second phase, such as feature-negative and differential conditioning. The effect persisted despite a large number of extinction trials (three times the number required to eliminate spontaneous recovery). Reinstatement tests also produced no evidence that US-alone presentations reinstated extinguished responding in the NMR paradigm (Napier et al., 1992, Experiment 1). Napier et al. interpreted their results with a model that formally captured the idea that residual excitatory strength was available to groups that had been through conditioning and extinction (Kehoe, 1988).

A recent fear-conditioning study has also found evidence consistent with rapid reacquisition (McAllister \& McAllister, 1994). Using the acquired drive method (acquisition of a hurdle-jumping response to escape fear conditioned to contextual cues), McAllister and McAllister found that reconditioning of fear following $30 \mathrm{~h}$ of extinction exposure to the context occurred more rapidly than did conditioning in a group analogous to a rest control. However, the result was not obtained in three earlier experiments that used less extinction (McAllister \& McAllister, 1994, Experiments 1-3). It is not clear why more extinction should produce the result when less extinction should be more likely to do so (e.g., Kehoe, 1988).

Given that reacquisition can occur both rapidly and slowly, our overall goal was to determine what conditions produce the two results. One possibility, suggested by Napier et al. (1992), is that different conditioning preparations have intrinsic differences that yield rapid or slow reacquisition. Our initial goal, then, was to characterize reacquisition in a preparation that had not been studied before. We chose an appetitive conditioning method that is widely used in the conditioning literature (e.g., Balleine \& Dickinson, 1991; Bouton \& Ricker, 1994; Delamater, 1995; Farwell \& Ayres, 1979; Hall \& Channell, 1985; Kaye \& Mackintosh, 1990; Pearce \& Redhead, 1995). Food pellets served as the US, and the rat's entries into the magazine to which the pellets were delivered served as the conditioned response. The magazineentry response is often described as a Pavlovian CR, but since obtaining the pellet is directly contingent on the response, the response presumably also has an instrumental component. Indeed, when magazine approach is put on an omission schedule (so that responding on a trial leads to the omission of a reinforcer that is otherwise delivered; Holland, 1979), the response is acquired and maintained (suggesting some Pavlovian control) but is also weaker than that in a control group that receives the same CS-US pairings without the omission contingency (suggesting an instrumental component). As an instrumental response, magazine entry is probably best described as a partially reinforced discriminated operant; many magazine entries go unreinforced during both the $\mathrm{CS}$ and the intertrial interval. The complexity of the magazine-entry response will require some caution in interpretation. But there now appear to be many similarities between instrumental and Pavlovian learning (e.g., Mackintosh, 1983; Rescorla, 1987). And reacquisition after instrumental extinction, like Pavlovian extinction, is often assumed to occur rapidly (e.g., Bullock \& Smith, 1953).

The present experiments obtained results suggesting that both slow and rapid reacquisition can occur in the magazine-entry preparation. They also identified sequential learning (e.g., Capaldi, 1994) as a variable that contributes to which of these results is obtained. During conditioning, reinforced trials may be associated with other reinforced trials. When reinforced trials are presented following extinction, they may renew responding on subsequent trials.

\section{EXPERIMENT 1}

We designed Experiment 1 as an initial investigation of reacquisition in our appetitive conditioning preparation. In Experiment 1A, we examined the rate of reacquisition in a group that had received conditioning and extinction with a tone CS (Group $R$ ) relative to conditioning in several control groups. The first of these was a learningexperienced control similar to the one used by Bouton and Swartzentruber (1989); this group received an iden- 
tical treatment with a different CS (a light-off stimulus) during conditioning and extinction (Group L). A second control received equivalent exposure to the US during the conditioning phase but no pairings of the US with any stimulus (Group U). The last group (Group C) was a rest control that received no stimulus presentations during the conditioning or extinction phases (cf. Bouton, 1986; Napier et al., 1992). During a final reacquisition phase, all groups received tone-food pairings. In Experiment 1B, we doubled the number of extinction trials and compared a reacquisition group with a learning-experienced control.

\section{Experiment 1A}

\section{Method}

Subjects. The subjects were 32 male Wistar rats bred at the University of Vermont. They were approximately 120 days old at the start of the experiment and were individually housed in suspended stainless steel cages in a room maintained on an 18:6-h light:dark cycle. The experiment was conducted on consecutive days during the light portion of the cycle. The rats were food deprived to $80 \%$ of their free-feeding weights and maintained at that level throughout the experiment.

Apparatus. Two sets of four Skinner boxes, housed in soundattenuation chambers and located in two separate rooms, were used. These two sets of boxes have provided different contexts in other studies, but were not so used in the present experiment. Each box in one set measured $26 \times 25 \times 19 \mathrm{~cm}$. The front, back, and one side wall were made of aluminum; the ceiling and other side wall were made of clear plastic. The floor consisted of tubular steel bars $16 \mathrm{~mm}$ in diameter, spaced $3.2 \mathrm{~cm}$ center to center, and mounted perpendicular to the front wall. On the front wall of each box, $1 \mathrm{~cm}$ above the floor, was a recessed $4 \times 4 \mathrm{~cm}$ food cup. A $2.5 \times 2.5 \mathrm{~cm}$ lever protruded from the front wall $5 \mathrm{~cm}$ above the floor and $1 \mathrm{~cm}$ to the right of the food cup. The subjects were placed in the boxes through a door in the right wall.

Each box in the second set measured $24 \times 22 \times 18 \mathrm{~cm}$. The front and back walls were aluminum, while the ceiling and side walls were clear plastic with vertical black stripes $2 \mathrm{~cm}$ wide and $2.5 \mathrm{~cm}$ apart. The floor consisted of stainless steel bars $3 \mathrm{~mm}$ in diameter, spaced $1.5 \mathrm{~cm}$ from center to center, and mounted parallel to the front wall. On the front wall of each box, $1 \mathrm{~cm}$ above the floor and centered $3.5 \mathrm{~cm}$ from the right wall, was a recessed $4 \times 4 \mathrm{~cm}$ food cup. A $4 \times 1 \mathrm{~cm}$ lever protruded from the front wall $5 \mathrm{~cm}$ above the floor and $6 \mathrm{~cm}$ to the left of the food cup. The subjects were placed in the boxes through the ceiling.

In both sets of boxes, illumination was provided by two $7.5-\mathrm{W}$ white incandescent bulbs mounted on the ceilings of the soundattenuation chambers, $25 \mathrm{~cm}$ above the floor. Two CSs were used in this study. One was the $30-\mathrm{sec}$ presentation of a $3000-\mathrm{Hz}$ tone ( $80 \mathrm{~dB}$ re $20 \mu \mathrm{N} / \mathrm{M}^{2}$ [A]) provided by a generator wired to identical speakers mounted in each chamber $25 \mathrm{~cm}$ above the box floor. Background noise was $65 \mathrm{~dB}$. The other CS consisted of the 30 -sec offset of the houselights, which produced complete darkness. The US consisted of two 45-mg food pellets (Traditional formula, P. J. Noyes, Lancaster, NH) delivered $0.2 \mathrm{sec}$ apart. Magazine entries were detected by photocells mounted within the magazines, just behind the plane of the wall of the Skinner boxes. The apparatus was controlled by computer equipment located in an adjacent room.

Procedure. Pretraining. Each subject first received $30 \mathrm{~min}$ of exposure to the box it was assigned to for the duration of the experiment. Food cups were baited with 4 food pellets prior to the start of each exposure session. On the following day, subjects received one $30-\mathrm{min}$ session of magazine training. Food cups were again baited with 4 pellets each. During the session, the subjects were trained to approach and eat from the food cup upon activation of the feeder mechanism. Approximately 25 pellets were delivered during each session.

Conditioning. The subjects then received 6 days of conditioning. On each day, there was one 90-min session. For Groups $R$ and $\mathrm{L}$, this session consisted of eight pairings of the 30-sec CS with the food US, with a mean intertrial interval (ITI) of $10 \mathrm{~min}$ (shortest ITI $=9 \mathrm{~min})$. Throughout, the offset of the CS coincided with the onset of the US. For Group R the CS was the tone (T), while for Group $L$ the CS was the light off (L). Group U received the same eight US presentations but no CS. Group C was merely placed in the apparatus for the entire $90 \mathrm{~min}$; no stimuli were presented. On Day 1, the first session for each group started with two nonreinforced presentations each of $\mathrm{T}$ and $\mathrm{L}$ alone in the sequence TLTL, with a mean ITI of 1 min.

Extinction. Following conditioning, the rats received 10 sessions of extinction. All sessions were $90 \mathrm{~min}$ in duration and occurred on consecutive days. For Groups R and L, the 30-sec CS was presented eight times alone with a mean ITI of $10 \mathrm{~min}$ during each session. The CSs presented were the same as those used during conditioning. Groups $\mathrm{U}$ and $\mathrm{C}$ were both placed in the apparatus for the 90 -min session, but no stimuli were presented. No food was presented during this phase.

Reacquisition. Following extinction, the rats received four more sessions of conditioning. All four groups received one 90 min session a day in which the 30 -sec tone CS was paired with food eight times, with a mean ITI of $10 \mathrm{~min}$.

Data analysis. Conditioned responding was measured by means of an elevation score of the form $e=c-p$, where $c$ represents the number of magazine entries made during the $30-\sec \mathrm{CS}$ and $p$ represents the number of magazine entries made during the $30 \mathrm{sec}$ immediately preceding the CS. Elevation scores were calculated for each trial and then converted to four-trial block averages prior to analysis. These scores were then analyzed by means of a mixed design analysis of variance (ANOVA), with group as a between subjects factor and block as a repeated measure. An identical ANOVA was used to analyze responding during the pre-CS period (prescores). A rejection criterion of $p<.05$ was used throughout. Post hoc comparisons were conducted using $t$ tests with a pooled error term and a Bonferroni adjustment for Type I error rate.

\section{Results}

Conditioning proceeded uneventfully, with Groups $\mathbf{R}$ and $L$ reaching asymptote by Day 5 . The data are summarized on the left side of Figure 1. A group $\times$ block ANOVA on the conditioning data revealed only a significant main effect of block $[F(11,154)=7.11]$; responding tended to increase over blocks. Neither the main effect of group $[F(1,14)=1.22]$ nor the group $\times$ block interaction $[F(11,154)=1.16]$ approached significance.

Extinction proceeded uneventfully as well (right portion of Figure 1). A group $\times$ block ANOVA carried out on the data revealed only a significant main effect of block $[F(19,266)=15.43]$. Neither the main effect of group nor the group $\times$ block interaction were significant $(F \mathrm{~s}<1)$.

Group comparisons during reacquisition. The data for each group during the reacquisition phase are presented in the top panel of Figure 2. All groups acquired conditioned responding at this time. Groups $\mathrm{R}$ and $\mathrm{L}$ appeared to differ, with Group $\mathrm{R}$ responding somewhat more on early trial blocks and somewhat less on later ones. Groups $\mathrm{U}$ and $\mathrm{C}$ were both somewhat slower than 




Figure 1. Mean elevation scores during conditioning and extinction in Experiment 1A.

Groups $R$ and $L$ in acquiring responding, with Group $U$ being slowest. A group $\times$ block ANOVA revealed a significant main effect of block $[F(7,196)=12.28]$ and a significant group $\times$ block interaction $[F(21,196)=2.88]$. The main effect of group was not significant $[F(3,28)=$ 2.78].

The interaction suggests that group differences existed only on certain trial blocks. Therefore, a series of five unplanned comparisons were conducted with an adjustment for error rate (which set $\alpha=.01$ ). These comparisons compared Group $R$ with each of the control groups separately on the blocks where the largest group differences appeared, and similarly compared Group L with each of the other controls. Group R did not differ significantly from Group L on Block $7[t(78)=-2.11]$, where the largest difference between these two groups appeared. However, Group R responded significantly more than Group $U$ on Block $4[t(78)=3.13]$ and more than Group $C$ on Block $3[t(78)=2.77]$. Group L also responded more than Group $U[t(78)=2.88]$ and Group C $[t(78)=2.68]$ on Block 5 .

Although Groups $\mathrm{R}$ and $\mathrm{L}$ failed to differ in the previous analysis, previous CER and taste-aversion experiments that found slow reacquisition had compared analogous groups by themselves. Therefore, an additional analysis was conducted using only these two groups. A group $\times$ block ANOVA revealed a significant main effect of block $[F(7,98)=6.98]$ and a significant group $\times$ block interaction $[F(7,98)=2.55]$. The main effect of group was not significant $(F<1)$. The interaction suggests that acquisition proceeded differently in Groups $\mathrm{R}$ and $L$. However, the largest difference between the groups (Block 7) was not reliable $[t(42)=-2.00]$.

The ANOVA carried out on prescores revealed no significant effects [largest $F(7,196)=1.87]$. Mean prescores for the reacquisition phase were $1.71,1.33,1.38$, and 1.29 for Groups R, L, U, and C, respectively.
Conditioning versus reconditioning. The upper panel of Figure 3 shows Group R's performance in both the original conditioning phase (Phase 1 ) and during reacquisition (Phase 3). A within-subject comparison of performance in these phases can complement our betweensubjects analysis of reacquisition, although it does confound conditioning history of the CS with other variables, such as prior experience with the reinforcer, the apparatus, and the food-deprivation schedule. As Figure 3 suggests, responding was clearly greater during reconditioning. A within-subject phase $\times$ block ANOVA revealed a significant main effect of phase $[F(1,7)=$ 16.73]. The ANOVA also revealed a significant main effect of block $[F(7,49)=4.86]$ and a significant phase $\times$ block interaction $[F(7,49)=5.41]$.

A similar ANOVA on prescores revealed a significant main effect of block $[F(7,49)=2.53]$. Prescores increased slightly over blocks, regardless of phase. The main effect of phase approached significance, but was not reliable $[F(1,7)=5.15, p=.06]$, and the phase $\times$ block interaction was not significant $[F(7,49)=1.00]$. Mean prescores were 1.34 and 1.71 during conditioning and reacquisition, respectively.

\section{Experiment 1B}

\section{Method}

Subjects and Apparatus. The subjects were 16 male Wistar rats of the same stock as those used in Experiment 1A. They were approximately 160 days old at the start of the experiment, and were housed and maintained as in Experiment 1A. One subject in Group $L$ became sick during the course of the experiment and was removed, leaving 7 subjects in that group. The apparatus was the same as that used in Experiment $1 \mathrm{~A}$.

Procedure. Pretraining. Box exposure and magazine training were carried out as in Experiment 1A. Approximately 28 pellets were delivered during each magazine training session.

Conditioning. Following magazine training, the subjects received eight sessions of conditioning. Each rat received two 


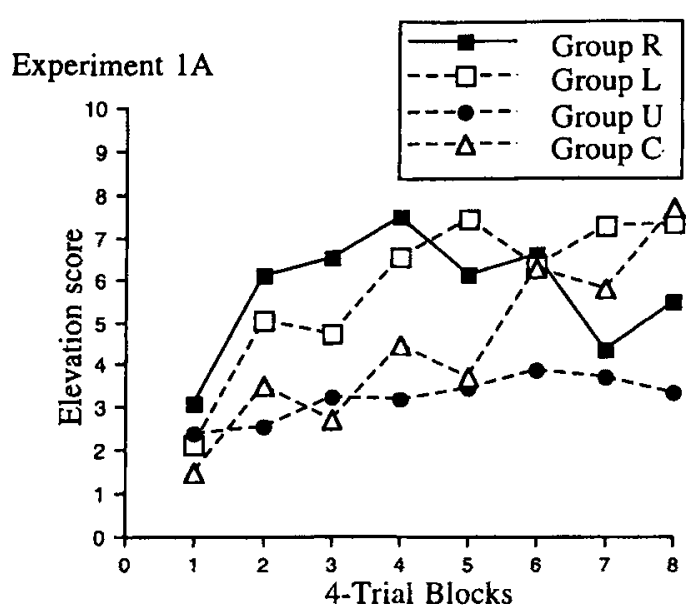

Experiment 1B

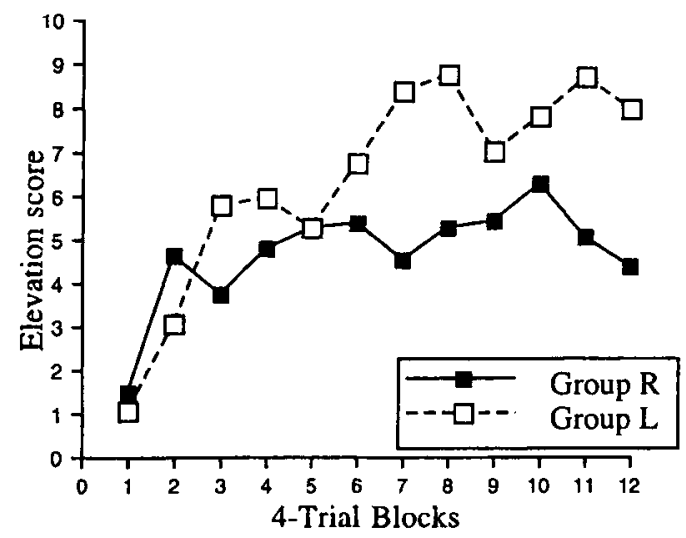

Figure 2. Mean elevation scores during reacquisition in Experiments $1 \mathrm{~A}$ (upper panel) and $1 \mathrm{~B}$ (lower panel).

90-min sessions on each day. Each session contained eight pairings of the 30-sec CS with the food US, with a mean ITI of $10 \mathrm{~min}$. For Group R, the CS was T; for Group L, it was L. On Day 1, the first session for each group started with two presentations each of $T$ and $L$ alone in the sequence TLTL, with a mean ITI of $1 \mathrm{~min}$.

Extinction. Following conditioning, the rats received 20 sessions of extinction. As in conditioning, there were two 90 -min sessions on each day. In each session, the 30-sec CS was presented eight times alone with a mean ITI of 10 min. A total of 160 extinction trials were given. The CSs presented were the same as those used during conditioning. No food was presented during this phase.

Reacquisition. Following extinction, the rats received six more sessions of conditioning. Both groups received two 90 -min sessions a day in which the $30-\mathrm{sec}$ tone CS was paired with food eight times, with a mean ITI of $10 \mathrm{~min}$. Data analysis was carried out as in Experiment 1.

\section{Results}

Conditioning proceeded uneventfully; during the final four-trial block of acquisition, Groups $R$ and $L$ had mean elevation scores of 5.28 and 5.14, respectively. A group $\times$ block ANOVA revealed only a significant main effect of block $[F(15,195)=10.55]$. Neither the main effect of group $[F(1,13)=2.56]$ nor the interaction $[F(15,195)=$ 1.52] were significant. During extinction, responding appeared to extinguish more slowly in Group L than in
Group R; the mean elevation scores for the phase were 0.63 and 0.33 for Groups $L$ and $R$, respectively. A group $\times$ block ANOVA revealed a significant main effect of group $[F(1,13)=4.72]$ and a significant main effect of block $[F(39,507)=6.42]$. The interaction was not significant $(F<1)$. Although Group L appeared to extinguish more slowly, this difference had disappeared by the last four sessions, where Groups $\mathrm{L}$ and $\mathrm{R}$ had mean elevation scores of 0.14 and 0.17 , respectively. Four unplanned comparisons (at $\alpha=.0125$ ) between the two groups on the first block of each of the last four sessions revealed no significant differences $[t s(405)<1]$.

Group comparison during reacquisition. The focal data from reacquisition are presented in the lower panel of Figure 2. During this phase, both groups received conditioning with the tone. The groups initially did not differ, but Group R showed less responding than Group L over blocks. This description was confirmed by statistical analysis. A group $\times$ block ANOVA revealed a significant main effect of block $[F(11,143)=11.93]$ and a significant group $\times$ block interaction $[F(11,143)=$ 3.33]. The main effect of group was not significant $[F(1,13)=2.33]$. Comparisons (at $\alpha=.0167$ ) between groups on Blocks 7, 11, and 12, where the largest group
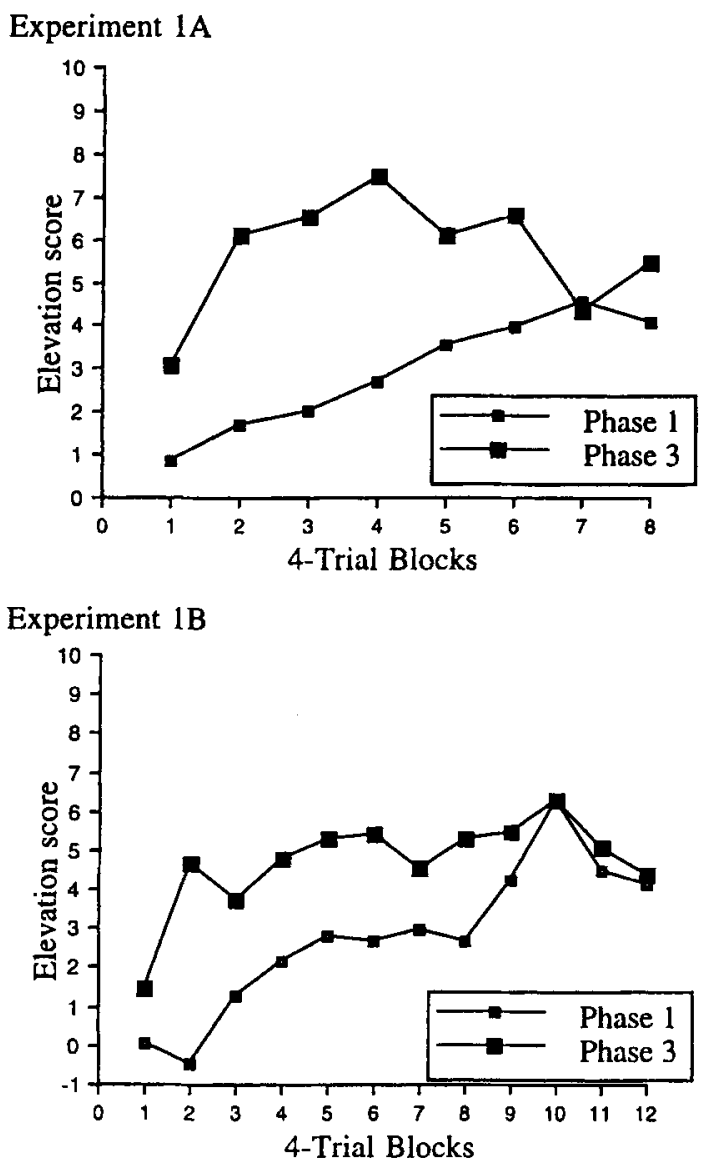

Figure 3. A comparison of mean elevation scores for Group $R$ during conditioning and reacquisition in Experiments $1 \mathrm{~A}$ (upper panel) and $1 B$ (lower panel). 
differences appeared, revealed less responding in Group $\mathrm{R}$ than in Group L on Block $7[t(35)=-2.68]$ and Block 11 $[t(35)=-2.53]$ but not on Block $12[t(35)=-2.50]$.

The ANOVA on prescores revealed only a significant main effect of block $[F(11,143)=3.67]$; responding during the pre-CS period increased somewhat and then decreased over blocks. All other effects were not significant $(F \mathrm{~s}<1)$. Mean prescores for the reacquisition phase were 1.27 and 0.90 for Groups R and L, respectively.

Conditioning versus reconditioning. The lower panel of Figure 3 presents a comparison of Group R's performance during reacquisition and the original conditioning phase. A phase $\times$ block ANOVA revealed a significant main effect of phase $[F(1,7)=12.09]$, a significant main effect of block $[F(11,77)=7.80]$, and a significant phase $\times$ block interaction $[F(11,77)=2.46]$. Again, conditioning occurred more rapidly in the reacquisition phase than in the original conditioning phase.

A similar ANOVA on prescores revealed no significant effects [largest $F(11,77)=1.19$ ]

\section{Discussion}

The results of Experiments $1 \mathrm{~A}$ and $1 \mathrm{~B}$ indicate that reacquisition in the appetitive method can be rapid when compared with "learning-naive" controls (Groups U and $\mathrm{C}$ in Experiment $1 \mathrm{~A}$ ) and with the original conditioning phase. However, in Experiment 1B, performance during reacquisition was impaired relative to that in Group $\mathrm{L}$, a learning-experienced control (a related group $\times$ session interaction was obtained in Experiment 1A). Group L itself appeared to show a "learning-to-learn" effect analogous to that previously observed in NMR conditioning (e.g., Kehoe \& Holt, 1984; Kehoe, Morrow, \& Holt, 1984). That is, previous conditioning (and extinction) with the light-off CS facilitated conditioning with the tone relative to learning-naive controls. Somewhat paradoxically, a learning-to-learn effect was less evident in a reacquisition group that had received all three phases with the same CS. That result might implicate an interference mechanism in addition to the learning-to-learn mechanism.

Although the fact that Group R was lower in responding than Group $L$ later in the phase indicates some interference by prior conditioning and extinction with the same CS, it is worth noting that, in CER, the interference effect is observed starting early in reacquisition. Instead of acquiring responding more slowly, Group R appeared to approach a lower asymptote (see especially Experiment 1B). Theories that predict slow reacquisition would actually expect group differences to occur early, rather than late, in the phase (Bouton, 1993; Pearce \& Hall, 1980; Wagner, 1981).

\section{EXPERIMENT 2}

In Experiment 2, we conducted reacquisition with a partial reinforcement procedure that intermixed nonreinforced trials among the reinforced trials. This sort of procedure was used by Bouton (1986) and Bouton and Swartzentruber (1989), who obtained slow reacquisition.
According to Bouton's (1993) retrieval view, presentation of nonreinforced trials during reacquisition might help retrieve a memory of extinction for a reacquisition group and thus cause reacquisition to occur slowly.

This analysis resembles Capaldi's (e.g., 1994) welldeveloped sequential theory. It implies that the outcome of a given trial can serve as a signal (or retrieval cue) for the outcome of the next trial. Thus, during conditioning, reinforced trials might come to signal that the next trial will be reinforced, while during extinction nonreinforced trials might come to signal that the next trial will be nonreinforced. This view implies a particular pattern of responding during reacquisition with partial reinforcement. Following conditioning and extinction, responding on a trial after a reinforced trial should be high, while responding following a nonreinforced trial should be low. Since reacquisition was conducted with a partial reinforcement procedure in this experiment, we could test this prediction. A retrieval view emphasizing the role of previous trials predicts more responding after reinforced than after nonreinforced trials.

We used a learning-naive control similar to Group $\mathrm{U}$ in Experiment 1A (as opposed to Group C) so that the control would have had equal exposure to the US. This group also received conditioning in the third phase with a partial reinforcement procedure. Here one might also expect higher responding on trials following reinforced trials, if only because associative strength might increase after each CS-US pairing. Clearly, any sequential learning effect in a reacquisition group would have to be greater than that observed in Group U.

We also performed a context switch following US exposure in order to reduce possible blocking by context conditioning (e.g., Randich, 1981). There is some evidence to suggest that this occurred in Experiment $1 \mathrm{~A}$, because in the final phase Group $U$ appeared to acquire conditioned responding more slowly than Group $\mathrm{C}$, the control that had not received US exposure in Phase 1.

\section{Method}

\section{Subjects}

The subjects were 16 male Wistar rats from the same stock as in the previous experiments, and were maintained and housed as before. They were approximately 100 days old at the start of the experiment.

\section{Apparatus}

The apparatus was the same as in Experiments $1 \mathrm{~A}$ and $1 \mathrm{~B}$, except that a dish containing $10 \mathrm{ml}$ of Heinz distilled white vinegar (H. J. Heinz Co., Pittsburgh) was placed in each sound-attenuation chamber of the first set of boxes in order to provide a distinctive scent cue; a dish containing $1 \mathrm{~g}$ of Vick's Vaporub (RichardsonVicks, Inc., Shelton, CT) was similarly placed in each soundattenuation chamber of the second set of boxes. In the present experiment, the two sets of boxes, which provided the different contexts, were fully counterbalanced.

\section{Procedure}

Pretraining. Box exposure and magazine training were carried out as in Experiment 1, with the exception that each subject received one session in Context $\mathrm{A}$ (the conditioning context) and one in Context B (the US exposure context). Sessions in different 
contexts were conducted on the same day, although box exposure and magazine training were conducted on separate days, as before. Approximately 20 pellets were delivered during each of the magazine training sessions.

Conditioning. The subjects then received 8 days of conditioning. On each day, there were two 90 -min sessions, one in Context $\mathrm{A}$ and one in Context B. For Group R, sessions in Context A consisted of eight pairings of the $30-\mathrm{sec}$ tone CS with the food US, with a mean ITI of $10 \mathrm{~min}$. Sessions in Context B for this group consisted only of exposure to the apparatus. For Group U, sessions in Context A consisted of exposure to the apparatus, while sessions in Context $B$ consisted of eight presentations of the US alone, with a mean ITI of $10 \mathrm{~min}$. As in the preceding experiments, on Day 1 all sessions started with two presentations of the tone alone, with a mean ITI of $1 \mathrm{~min}$, so that conditioning began with a nonnovel $\mathrm{CS}$ in all groups. Group $\mathrm{R}$ received sessions in the order ABBA, while Group $U$ received them in the order BAAB.

Extinction. Following conditioning, the rats received 20 sessions of extinction in Context A. There were two 90 -min sessions in Context A each day. For Group R, each session consisted of eight presentations of the tone alone, with a mean ITI of $10 \mathrm{~min}$. For Group U, these sessions consisted only of exposure to the apparatus. No food was presented during this phase.

Reacquisition. Following extinction, the rats received 3 more days (six sessions) of conditioning in Context $\mathrm{A}$. Both groups received two 90 -min sessions a day in which the $30-\mathrm{sec}$ tone CS was paired with food four times and presented alone four times, with a mean ITI of $10 \mathrm{~min}$. Two trial sequences were used over the six sessions. In one sequence, reinforced $(\mathrm{R})$ and nonreinforced $(\mathrm{N})$ trials alternated (i.e., RNRNRNRN); in the other sequence, trials semialternated (i.e., RNRRNNRN). The order of presentation of the two sequences was ASSAAS, where A refers to alternating and $S$ refers to semialternating.

\section{Results}

By the end of conditioning, rats in Group $\mathrm{R}$ were responding well to the CS; the mean elevation score in the last four-trial block was 7.72. A one-way repeated measures ANOVA was conducted for Group R, and it revealed a significant main effect of four-trial block $[F(15,105)=4.21]$. Extinction also proceeded without incident, with rats in Group $\mathrm{R}$ showing little or no spontaneous recovery by Day 4 . By the last session of extinction, Groups $\mathrm{R}$ and $\mathrm{U}$ had mean elevation scores of 0.00 and -1.88 , respectively. A trial-block ANOVA revealed a significant main effect of block $[F(39,273)=$ 7.71].

Reacquisition data are presented in Figure 4. During reacquisition, Groups $R$ and $U$ initially did not differ, but over trials, $R$ responded more than $U$. A group $\times$ block ANOVA carried out on elevation scores revealed a significant main effect of group $[F(1,14)=7.20]$, a significant main effect of block $[F(11,154)=8.10]$, and a significant group $\times$ block interaction $[F(11,154)=3.45]$. Although responding overall was higher in Group $R$, the groups did not differ initially and toward the end of reacquisition. This was confirmed with four post hoc comparisons (at $\alpha=.0125$ ). On Block 1 , Groups $R$ and $U$ did not differ $[t(44)=0.53]$. However, on Blocks 6 and 10 , they did $[t s(44) \geq 3.44]$. By Block 12 , the groups no longer differed $[t(44)=1.86]$.

The ANOVA on prescores revealed only a significant main effect of block $[F(11,154)=3.13]$. Neither the main effect of group $[F(1,14)<1]$ nor the interaction $[F(11,154)=1.10]$ was significant. Prescores increased somewhat and then decreased over blocks, but did not differ between groups. Mean prescores were 1.64 and 1.55 for Groups $R$ and $U$, respectively.

Figure 5 presents the responding by Groups $R$ and $U$ on trials that followed reinforced versus nonreinforced trials. A group $\times$ trial type $\times$ session ANOVA was conducted on the elevation scores. The ANOVA revealed a significant main effect of group $[F(1,14)=7.20]$, a significant main effect of session $[F(5,70)=12.59]$, and a significant group $\times$ session interaction $[F(5,70)=3.23]$ (these effects correspond to the preceding analysis of reacquisition). In addition, there was a significant main effect of trial type $[F(1,14)=5.48]$ and, most importantly, a significant trial type $\times$ group interaction $[F(1,14)=$ 17.62]. While responding overall was higher following reinforced than following nonreinforced trials, this effect was confined to Group $R$. The session $\times$ trial type interaction was significant $[F(5,70)=2.70]$, but more importantly, the session $\times$ trial type $\times$ group interaction was also significant $[F(5,70)=2.37]$. Initially, for Group $\mathrm{R}$ only, responding was higher following reinforced trials than following nonreinforced trials, but, over sessions, this difference disappeared. This was confirmed with simple comparisons (at $\alpha=.0125$ ). In Group $\mathrm{R}$ there were significant differences between trial types during the first two sessions $[t \mathrm{~s}(82)>4.44]$, but in Group $\mathrm{U}$ there were no such differences $(t \mathrm{~s}<1)$.

A similar analysis was conducted on prescores. The ANOVA revealed significant main effects only of session $[F(5,70)=5.63]$ and trial type $[F(1,14)=5.77]$. Responding during the preperiod was somewhat higher following reinforced trials than following nonreinforced trials, where the respective means were 1.74 and 1.54 for Group R and 1.81 and 1.28 for Group U. The fact that this effect did not vary with group [group $\times$ trial type interaction, $F(1,14)=1.15]$ is interesting, given that the



Figure 4. Mean elevation scores during reacquisition in Experiment 2. 




Figure 5. Mean elevation scores during reacquisition in Experiment 2. Here data for Groups $R$ and $U$ are broken down into trials following reinforced trials ( $r$ ) and trials following nonreinforced trials (n).

elevation scores did vary with group on these trials. This suggests that the aftereffects of a reinforced trial were experienced in both groups (higher prescores), but only in Group $\mathrm{R}$ did a reinforced trial signal reinforcement on the next trial.

\section{Discussion}

Reacquisition again occurred rapidly compared with a learning-naive control, even when a partial, rather than continuous, reinforcement procedure was used. However, closer analysis revealed that high responding in Group R was initially confined to trials following reinforced, rather than nonreinforced, trials. This result is consistent with a retrieval view, especially one emphasizing the retrieval role of individual trials (e.g., Capaldi, 1994). The fact that these differences in responding did not last beyond the first two sessions may be due to the change in contingencies between reinforced and nonreinforced trials and the trial types that followed them. That is, during reacquisition, nonreinforced trials now tended to signal reinforced trials and reinforced trials tended to signal nonreinforced trials, reversing what might have been learned during extinction and conditioning.

Responding following nonreinforced trials was not lower in Group $\mathrm{R}$ than in Group U. This could present a problem for the retrieval view. If, for Group $R$, nonreinforced trials were signaling nonreinforcement on the next trial, then responding following nonreinforced trials should have been lower in this group than in Group $U$, for whom no such signaling should occur. One possibility is that if retrieval of an extinction memory is more susceptible to retrieval failure (Bouton, 1993), it may be more difficult to retrieve the memory of nonreinforcement than of reinforcement in Group R. Whatever the merit of this idea, it does appear that the rapid reacquisition observed in the present method is initially restricted to trials that follow reinforced trials, as a retrieval view or sequential theory predicts.

\section{EXPERIMENT 3}

One purpose of Experiment 3 was to replicate the sequential learning effect observed in Experiment 2. Another purpose was to further explore the learning-tolearn effect that was observed in Experiment $1 \mathrm{~A}$. In that experiment, conditioning and extinction with the lightoff CS facilitated conditioning of the tone CS in the final phase. This sort of result is captured by the model presented by Kehoe (1988), which states that conditioning and extinction with any stimulus results in residual excitation which may transfer to reconditioning or conditioning of another stimulus. An alternative possibility, however, is that the sequential learning effect identified in Experiment 2 may contribute to this result as well. Following conditioning, the occurrence of the US might potentially serve as a general signal of reinforcement, regardless of the identity of the CS. If so, we might likewise observe enhanced responding following reinforced trials in a learning-experienced control group. In Experiment 3, we therefore compared responding on trials after reinforced and nonreinforced trials in Phase 3 in a learning-experienced control (Group L), a reacquisition group, and a rest control.

\section{Method}

\section{Subjects}

The subjects were 24 female Wistar rats obtained from Charles River, Canada. (The rats in Experiments $1 \mathrm{~A}-\mathrm{B}$ and 2 were derived from this stock.) They were approximately 90 days old at the start of the experiment and were housed and maintained as before. During the course of the experiment, 3 of the animals became sick ( 1 from each group) and were removed, leaving 7 animals in each group.

\section{Apparatus}

The apparatus was the same as that in Experiments 1 and 2, with the exception that the two Noyes food pellets that served as the US were delivered $0.4 \mathrm{sec}$ apart.

\section{Procedure}

Pretraining. Box exposure and magazine training were carried out as in Experiments 1 and 2. Approximately 18 pellets were delivered during each of the magazine training sessions.

Conditioning. The subjects received eight sessions of conditioning, one per day. For Group R, the sessions consisted of eight pairings of the $30-\mathrm{sec}$ tone with the food US, with a mean ITI of $10 \mathrm{~min}$. For Group L, the sessions consisted of eight pairings of the 30 -sec light off with the US, with a mean ITI of $10 \mathrm{~min}$. For Group C, these sessions consisted only of exposure to the apparatus; no stimuli were presented. For all groups, the first session started with two nonreinforced presentations each of the tone and light off, in the order TLTL.

Extinction. Following conditioning, the rats received 20 sessions of extinction, 1 per day. For Group R, these sessions consisted of eight presentations of the tone alone with a mean ITI of 10 min. For Group L, the sessions consisted of eight presentations 
of the light off alone with a mean ITI of $10 \mathrm{~min}$. Group $\mathrm{C}$ received only exposure to the apparatus during these sessions. No food was presented during this phase.

Reacquisition. Following extinction, the rats received three more sessions of conditioning, again one per day. For all groups, the tone was paired with food four times and was presented alone four times with a mean ITI of 10 min during these sessions. In each session, reinforced and nonreinforced trials alternated (i.e., RNRNRNRN).

\section{Results}

Conditioning proceeded uneventfully, with Groups $\mathrm{R}$ and $L$ reaching mean elevation scores of 5.93 and 5.75 , respectively, during the final four-trial block. A group $X$ four-trial block ANOVA revealed a significant main effect only of block $[F(15,210)=2.09]$. Neither the main effect of group nor the interaction were significant $[F(1,14)=1.97$ and $F(15,210)=0.93$, respectively $]$.

Extinction proceeded uneventfully as well, with rats showing little or no responding by Session 7. A group $\times$ block ANOVA revealed a significant main effect only of block $[F(39,468)=1.58]$. Neither the main effect of group nor the interaction were significant $\left(F_{\mathrm{S}}<1\right)$.

The data from reacquisition are presented in Figure 6. During reacquisition, Group $\mathrm{R}$ once again acquired responding more rapidly than Group $\mathrm{C}$ but more slowly than Group L. A group $\times$ block ANOVA revealed a main effect of group $[F(2,18)=5.17]$ and a main effect of block $[F(5,90)=9.57]$. The interaction was not significant $[F(10,90)=1.15]$. The main effect of group was explored with unplanned comparisons (at $\alpha=.017$ ). These comparisons revealed that Group $\mathrm{R}$ showed less overall responding than Group $\mathrm{L}[t(18)=-3.19]$, that Group $\mathrm{R}$ showed more responding than Group $\mathrm{C}$ $[t(18)=4.64]$, and that Group L showed more responding than Group $\mathrm{C}[t(18)=7.83]$. Although responding in Group $L$ seems unusually high at the outset, the first trial elevation score in this group was only -0.29 .

The ANOVA on prescores revealed a significant main effect only of block $[F(5,90)=3.82]$. Prescores increased slightly and then decreased over blocks. Neither the main effect of group nor the interaction were significant $[F(2,18)=1.24$ and $F(10,90)=0.96$, respectively] . Mean prescores for Groups $\mathrm{R}, \mathrm{L}$, and $\mathrm{C}$ were 1.86, 2.91, and 2.15 , respectively.

Figure 7 presents responding of each group on the trials following reinforced versus nonreinforced trials. A group $\times$ trial type $\times$ session ANOVA revealed significant main effects of group and session as in the previous analysis $[F(2,18)=5.17$ and $F(2,36)=11.06$, respectively]. The interaction of these factors was not significant $[F(4,36)=1.39]$. There was a significant main effect of trial type $[F(1,18)=11.46]$, indicating that responding was higher overall on trials following reinforced trials. None of the interactions involving trial type were significant $(F \mathrm{~s} \leq 1.22)$.

Because we had an a priori interest in which of the groups showed differentiation between trial types, trial type $\times$ session ANOVAs were conducted for each group

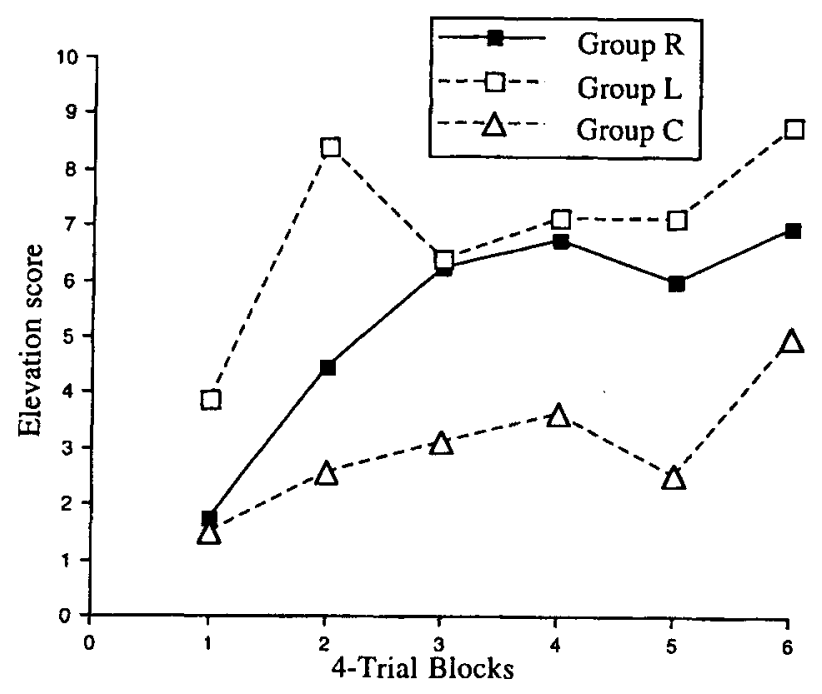

Figure 6. Mean elevation scores during reacquisition in Experiment 3.

separately, using the error terms from the overall analysis. For Group R, this analysis revealed both main effects of session $[F(2,36)=9.60]$ and trial type $[F(1,18)=$ 7.14]. For Groups L and C, however, there were no significant effects in these analyses [largest $F(1,18)=$ 3.89]. Further contrasts in Group $L$ on Sessions 1 and 2 revealed no differences in responding following reinforced versus nonreinforced trials $[t s(24) \leq 1.36]$. Thus, Group R, but neither Group L nor Group C, gave evidence of the sequential learning effect. The idea that facilitated responding in Group $\mathrm{L}$ was more than a result of sequential learning was further supported by ANOVAs comparing the groups' responding after reinforced and nonreinforced trials. Responding was greater in Group $L$ than in Group C following both reinforced $[t(18)=3.15]$ and nonreinforced $[t(18)>2.74]$ trials; facilitation was not dependent on trial type. In contrast, there was more responding in Group $\mathrm{R}$ than in Group $\mathrm{C}$ following reinforced trials $[t(18)=2.14, .025<p<.05]$ but not following nonreinforced trials $[t(18)=1.34]$. The facilitation effect for Group R, but not Group L, was thus demonstrably confined to trials following reinforced trials.

A group $\times$ trial type $\times$ session ANOVA on the prescores revealed a significant main effect of session $[F(2,36)=4.75]$. However, the three-way interaction was also significant $[F(4,36)=2.80]$. No other effects were significant in this analysis [largest $F(4,36)=1.56$ ]. Following reinforced trials, mean prescores for Groups $\mathrm{R}, \mathrm{L}$, and $\mathrm{C}$ were $1.64,2.63$, and 1.75 , respectively; following nonreinforced trials, they were $2.07,3.19$, and 2.15 , respectively. The three-way interaction suggests that differences in trial types existed for some groups only on some sessions. Therefore, separate trial type $X$ session ANOVAs were carried out for each group separately, as in the analysis of elevation scores. The only significant effects were a main effect of session for Group $L$ 


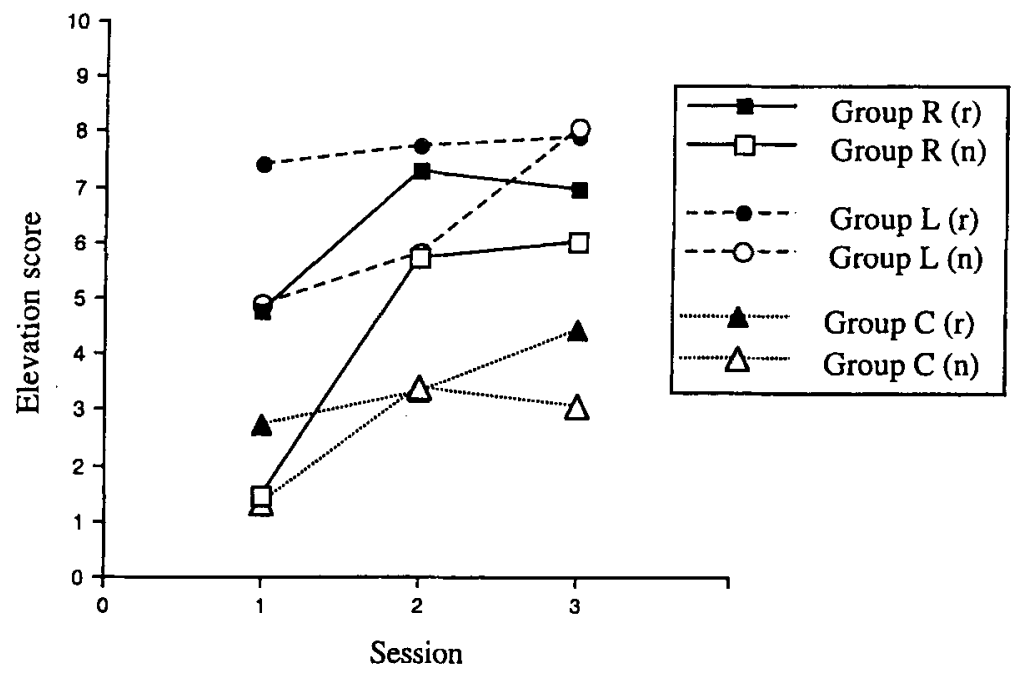

Figure 7. Mean elevation scores during reacquisition in Experiment 3. Here data are broken down into trials following reinforced trials (r) and trials following nonreinforced trials (n).

$[F(2,36)=4.64]$ and a trial type $\times$ session interaction for Group C $[F(2,36)=4.95]$. The interaction in Group C was further explored using simple comparisons (at $\alpha=$ $.017)$. These revealed only a small nonsignificant effect of trial type on Session $3[t(31)=-2.05, p=.05]$. Therefore, it can be concluded that there were no problematic differences in prescores.

\section{Discussion}

As in the previous experiments, reacquisition again occurred rapidly as compared with the learning-naive control (Group C) but was slow as compared with the learning-experienced control (Group L). In this experiment, the interference effect appeared early in the third phase, as would be expected given the results of previous studies (Bouton, 1986; Bouton \& Swartzentruber, 1989). This result is again consistent with the idea that two mechanisms influence reacquisition rate: a savings mechanism that allows groups that have been through conditioning and extinction with any CS to respond more in Phase 3 than groups that have not, and an interference mechanism that produces less of this effect in a reacquisition group than in a learning-experienced control group.

While responding was greater on trials following reinforced trials than on trials following nonreinforced trials, further analyses determined that this effect was confined to Group R. This result replicates the main result of Experiment 2, and continues to suggest that sequential learning plays a role in producing rapid reacquisition. In contrast, Group L did not show a reliable differentiation. It is possible that the present experiment was not powerful enough to detect an effect that might have existed in Group L; there was a clear numerical trend in the data. However, the fact that Group L's responding was significantly facilitated relative to Group C's following both nonreinforced and reinforced trials clearly indicates that the learning-to-learn effect was not restricted to trials following reinforced trials. Therefore, the learningto-learn effect evident in this preparation cannot be completely attributed to sequential learning.

\section{EXPERIMENT 4}

The purpose of the final experiment was to take a last look at whether reacquisition could occur slowly relative to a learning-naive control in this conditioning preparation. We decided to take advantage of an implication of the sequential learning view suggested by Experiments 2 and 3. Specifically, if rapid reacquisition was due in part to reinforced trials' signaling further reinforced trials, then one way to reduce the facilitative effect, and thus create an opportunity to view the interference effect, might be to drastically reduce the number of conditioning trials. With fewer conditioning trials, there would be fewer occasions for the animals to associate reinforced trials with other reinforced trials, and thus reacquisition might actually occur slowly as compared with a learning-naive control.

It is interesting to note that previous experiments showing slow reacquisition in CER and taste aversion have involved very few conditioning trials (eight in CER, and one-two in taste aversion). In Experiment 4 we therefore conducted conditioning with only eight trials. To obtain conditioning with this method in so few trials, we increased the magnitude of the food US from two to five $45-\mathrm{mg}$ food pellets and increased the ITIs from 10 to $20 \mathrm{~min}$. Trial-spacing parameters were identical to those of Bouton and Swartzentruber (1989) in the CER preparation. We hoped that by using only eight conditioning trials, acquisition of sequential learning would be minimized for Group $R$ and reacquisition would 
occur slowly compared with both a learning-experienced control and, for the first time, a rest control.

\section{Method}

\section{Subjects}

The subjects were 24 female Wistar rats obtained directly from Charles River, Canada. The rats were approximately 90 days old at the start of the experiment and were housed and maintained as before.

\section{Apparatus}

The apparatus was identical to that in Experiments 1 and 3, except that new, quieter feeders (Med Associates, Georgia, VT) replaced the original ones and Formula A/I pellets $(45 \mathrm{mg}, \mathrm{P}$. J. Noyes) were used. In this experiment, the food US consisted of five pellets delivered $0.4 \mathrm{sec}$ apart

\section{Procedure}

Pretraining. Box exposure and magazine training were carried out as in Experiments 1 and 3. Approximately 20 pellets were delivered during each magazine training session. Throughout the experiment, there was one session a day.

Conditioning. The subjects then received two sessions of conditioning. For Group R, the sessions consisted of four pairings of the $30-\mathrm{sec}$ tone with the five-pellet US. The mean ITI was $20 \mathrm{~min}$. Group L received the light-off CS instead of the tone. Group C received equal exposure to the apparatus but no stimuli.

Extinction. Following conditioning, the rats received 11 sessions of extinction. For Group $\mathrm{R}$, these sessions consisted of eight presentations of the tone alone with a mean ITI of $9.5 \mathrm{~min}$. Group $L$ received the light-off instead of the tone. On the last trial of the last extinction session, Group L received a nonreinforced preexposure of the tone instead of a light-off presentation (to eliminate the tone's novelty at the start of reacquisition; see Bouton \& Swartzentruber, 1989). Group C received only exposure to the apparatus during these sessions. No food was presented to any group during this phase.

Reacquisition. Following extinction, all rats received four sessions of tone conditioning. For all groups, the tone was paired with food two times and presented alone two times with a mean ITI of 20 min during these sessions. Reinforced and nonreinforced trials alternated (i.e., RNRN).

\section{Results}

Conditioning was successfully obtained in eight trials; Groups R and L had mean elevation scores of -0.44 and 0.81 on the first two-trial block and 4.44 and 3.50 on the final two-trial block of the phase. A group $\times$ two-trial block ANOVA carried out on all elevation scores from the phase revealed a significant main effect of block $[F(3,42)=7.25]$. Neither the main effect of group $[F(1,14)=1.26]$ nor the group $\times$ block interaction $[F(3,42)=2.08]$ were significant. A $t$ test comparing the mean of the final two-trial block with a hypothetical population mean of zero indicated significant responding in Group $\mathrm{R}[t(7)=4.64]$ and in Group $\mathrm{L}[t(7)=$ 5.24].

Extinction occurred fairly rapidly, with both groups showing little or no responding by Session 4 . A group $X$ block ANOVA revealed only a significant main effect of block $[F(43,602)=2.44]$. Neither the main effect of group $[F(1,14)=1.71]$ nor the group $\times$ block interaction $[F(43,602)=0.68]$ were significant.
The data from the reacquisition phase are presented in Figure 8. During reacquisition, Group $R$ acquired responding more slowly than Groups $L$ and $C$. A group $\times$ block ANOVA revealed a marginal but nonsignificant effect of group $[F(2,21)=3.17, p=.06]$, a significant effect of block $[F(7,147)=4.92]$, and a significant group $\times$ block interaction $[F(14,147)=1.92]$. Responding tended to increase over trials for all groups, but there were group differences on some trials. Three unplanned comparisons were conducted on the largest differences between Group $\mathrm{R}$ and each of the control groups and also between Group L and Group C (at $\alpha=.017$ ). These revealed that Group $R$ responded significantly less than Group C on Block $2[t(126)=-2.47]$ and significantly less than Group L on Block $5[t(126)=-3.37]$. Group L failed to differ from Group C on Block $5[t(126)=2.08]$.

A similar analysis carried out on prescores revealed no significant effects [largest $F(7,147)=1.74$ ]. Mean prescores for Groups $\mathrm{R}, \mathrm{L}$, and $\mathrm{C}$ were $1.18,1.56$, and 1.58 , respectively.

A group $\times$ trial type $\times$ session ANOVA was also performed on the data to assess possible sequential learning. This analysis revealed a marginal but nonsignificant effect of group $[F(2,21)=3.17, p=.06]$ and a significant effect of session $[F(3,63)=6.73]$, as in the previous analysis. However, the group $\times$ session interaction was not significant $[F(6,63)=1.57]$. There was a significant main effect of trial type $[F(1,21)=12.26]$, although none of the interactions involving trial type approached significance [largest $F(6,63)=1.21$ ]. The pattern indicates that the trial-type effect was evident regardless of group and session. The overall mean elevation scores following reinforced and nonreinforced trials, respectively, were 1.53 and 0.83 for Group R, 2.86 and 1.86 for Group L, and 3.12 and 2.21 for Group C.

As in Experiment 3, separate session $\times$ trial type ANOVAs were carried out for each group to determine

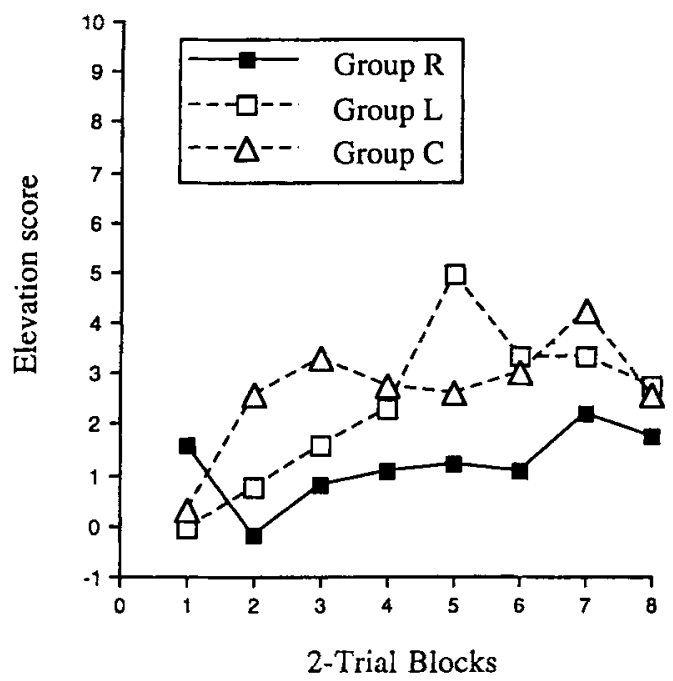

Figure 8. Mean elevation scores during reacquisition in Experiment 4 . 
if there was differential responding in each. These revealed that the main effect of trial type was significant only for Groups $\mathrm{L}$ and $\mathrm{C}[F(1,21)=5.37$ and $F(1,21)=$ 4.49 , respectively]. The trial type effect was not significant for Group $\mathrm{R}[F(1,21)=2.65]$. There were no significant interactions between session and trial type [largest $F(3,21)=1.78$ ].

A similar analysis carried out on prescores also revealed a main effect of trial type $[F(1,21)=5.21]$. However, it was in the opposite direction of that for elevation scores; responding was greater following nonreinforced trials (mean of 1.61) than following reinforced trials (mean of 1.27). Such a difference makes the previous analysis a conservative one, and is therefore not problematic.

\section{Discussion}

In this experiment, reacquisition occurred slowly as compared with the performance of both the learningexperienced and rest control groups. These results are consistent with the CER findings of Bouton (1986) and Bouton and Swartzentruber (1989), and they establish that reacquisition can occur more slowly in the appetitive method than in both learning-experienced and learningnaive controls. With the procedure used in this experiment, reacquisition was clearly slow, not fast. Interestingly, the learning-to-learn effect observed in Experiments $1 \mathrm{~A}$ and 3 was not observed in the present experiment, inasmuch as the learning-experienced control (Group L) failed to differ from the learning-naive control (Group C). This result may be consistent with other evidence suggesting that learning to learn may require training beyond a minimum number of conditioning trials (Schreurs \& Kehoe, 1987).

Slow reacquisition in this experiment is consistent with our prediction based on sequential theory: The smaller number of conditioning trials used here might have been insufficient to allow the rat to learn that reinforced trials predicted future reinforced trials. Therefore, performance during reacquisition was dominated by extinction performance retrieved by nonreinforced trials. It is worth noting, however, that the smaller number of conditioning trials used here also meant that the rats had had less experience retrieving pellets from the food magazine by the start of the reacquisition phase. That inexperience might have introduced a longer delay between the tone and the US (or the response and the reinforcer), although our informal observations suggested that, as in earlier experiments, the rats retrieved the pellets immediately upon their delivery during reacquisition. Another possibility is that the larger US used in this experiment was capable of generating more frustration on nonreinforced trials (see, e.g., Amsel, 1958; Wagner, 1969). Thus, anticipation of a large appetitive US might be especially effective at eliciting frustration behaviors that might compete with the magazine-entry response. And since Group R's tone had previously predicted food, Group $\mathrm{R}$ might be especially frustrated by trials on which it was nonreinforced. This possibility cannot be excluded by the present data. However, frustration the- ory cannot explain analogous results that have been reported with aversive, rather than appetitive, USs (Bouton, 1986; Bouton \& Swartzentruber, 1989; Hart et al., 1995). Those results, of course, can be handled by sequential theory.

The sequential learning effect evident in Experiments 2 and 3 was not evident here. Responding was generally higher on trials following reinforced than following nonreinforced trials, but further analysis revealed that this effect was not reliable in Group R. The greater responding following reinforced than following nonreinforced trials, particularly in Group C, was presumably due to the larger US used in this experiment, which could have caused large increments in associative strength following each reinforced trial. In fact, the increments must have been relatively large, given this US, because it produced significant conditioning in Phase 1 with only eight conditioning trials. The fact that we did not detect reliably greater responding following reinforced trials in Group $\mathrm{R}$ is consistent with the possibility that whatever mechanism causes slow reacquisition might be sufficiently strong to diminish those gains.

\section{GENERAL DISCUSSION}

In Experiments 1, 2, and 3, reacquisition was more rapid than acquisition in a rest control or a group that received an equal number of US presentations but no CS presentations prior to the third phase. In Experiments 1A and $1 \mathrm{~B}$, reacquisition was also more rapid than original acquisition in the original conditioning phase. These results clearly suggest that rapid reacquisition can occur in the appetitive magazine-entry conditioning preparation.

In contrast, in Experiments 1 and 3, reacquisition was impaired relative to acquisition in a learning-experienced control. Moreover, it was impaired relative to both a learning-experienced control and a rest control when conditioning was conducted with only eight trials and a partial reinforcement procedure was used during reacquisition (Experiment 4). These results suggest that both slow and rapid reacquisition can occur in the appetitive conditioning preparation.

Overall, then, the entire scope of previous results was obtained using a single conditioning method. This conclusion has at least two consequences. First, it strongly suggests that the different results obtained previously with different preparations were not necessarily due to inherent differences between the preparations themselves (cf. Napier et al., 1992). (Napier et al. had suggested that fast and slow reacquisition might be linked to consummatory and preparatory conditioning preparations, respectively.) Second, it is a difficult pattern for many existing theories to explain. For example, Kehoe's (1988) model does not predict slow reacquisition as compared with the performance of learning-experienced or rest controls under any circumstances. Conversely, although other models predict slow reacquisition after a large number of extinction trials, neither Pearce and Hall's (1980) model nor Wagner's (1981) SOP model predict 
the rapid reacquisition observed relative to the performance of learning-naive controls. Finally, without further refinement, the Bouton (1993) model cannot explain why a reacquisition group and a learning-experienced control both acquired conditioned responding faster than did learning-naive controls in Experiments 1 and 3.

One possibility implicated by the present results is that sequential learning is a critical determinant of reacquisition rate. The results of Experiments 2 and 3 indicated that responding during reacquisition was higher on trials following reinforcement than it was on nonreinforced trials in a reacquisition group; the same pattern was not evident in controls. Thus, reinforced trials appeared to signal subsequent reinforced trials in the reacquisition group. During conditioning, reinforced trials were presumably associated with other reinforced trials; similarly, during extinction, nonreinforced trials might have been associated with other nonreinforced trials. This sort of sequential learning could be implicit in many conditioning arrangements (e.g., Capaldi, 1994).

A sequential learning view actually predicts a complex set of outcomes in reacquisition experiments. If the acquisition of sequential learning depends on the number of acquisition trials, then the rate of reacquisition will be a function of the relative amount of training with each trial type. Rapid reacquisition would result from procedures involving a large number of initial reinforced trials, while slow reacquisition would result from procedures involving a large number of extinction trials. Slow reacquisition would also be more likely to occur when a partial reinforcement procedure is used during reacquisition, because the presence of nonreinforced trials would signal additional nonreinforced, as opposed to reinforced, trials.

These possibilities are generally consistent with the known facts of reacquisition. First, slow reacquisition does depend on extensive extinction training (Bouton, 1986). Second, rapid reacquisition appears to be limited to procedures involving extensive initial conditioning. In Experiments $1-3$ of the present study, where the reacquisition observed was rapid relative to that of learningnaive controls, the animals had received a large number $(\geq 48)$ of conditioning trials. In contrast, in previous CER and taste-aversion experiments, where slow reacquisition had been observed, there had been very few initial conditioning trials $(\leq 8)$. Furthermore, when appetitive conditioning was carried out in Experiment 4 with only eight trials, slow reacquisition was observed. Procedures that use many conditioning trials, then, will, due to strong associations among reinforced trials, be more likely to produce rapid reacquisition. This would be especially likely in the NMR paradigm (e.g., Napier et al., 1992), which requires hundreds of trials for conditioning with typical parameters. This view predicts that if numerous conditioning trials were delivered in CER or taste aversion, rapid reacquisition might be observed in these paradigms. It also predicts that if fewer conditioning trials were used in NMR conditioning, slow reacquisition would be observed.
One limitation of the sequential learning interpretation at present is that it does not explain why learningexperienced controls acquire conditioned responding more quickly than learning-naive controls-the learningto-learn effect (e.g., Kehoe \& Holt, 1984). There is a possible sequential learning explanation: Associations among reinforced trials learned with one CS might generalize to reinforced trials with a second CS. Thus, a learning-experienced group might learn that reinforced trials generally follow other reinforced trials, regardless of the CS. However, the results of Experiment 3 did not support this view; a learning-experienced group responded well in Phase 3 , but did not respond reliably more after reinforced than after nonreinforced trials. On the other hand, it is possible that the reinforced-trial signal generalized in such a group but that a nonreinforced trial signal did not. Such a possibility would explain why responding was high, but relatively undifferentiated, in the learning-experienced group. Though speculative, this possibility is consistent with other evidence suggesting that retrieval of extinction is relatively easy to disrupt with stimulus change (e.g., Bouton, 1993).

\section{REFERENCES}

AMSEL, A. (1958). The role of frustrative nonreward in noncontinuous reward situations. Psychological Bulletin, 55, 102-1 19.

Balleine, B., \& Dickinson, A. (1991). Instrumental performance following reinforcer devaluation depends upon incentive learning. Quarterly Journal of Experimental Psychology, 43B, 279-296.

Bouton, M. E. (1986). Slow reacquisition following extinction of conditioned suppression. Learning \& Motivation, 17, 1-15.

Bouton, M. E. (1991). Context and retrieval in extinction and in other examples of interference in simple associative learning. In L. Dachowski \& C. F. Flaherty (Eds.), Current topics in animal learning: Brain, emotion, and cognition (pp. 25-53). Hillsdale, NJ: Erlbaum.

Bouton, M. E. (1993). Context, time, and memory retrieval in the interference paradigms of Pavlovian learning. Psychological Bulletin, $114,80-99$.

Bouton, M. E., \& Bolles, R. C. (1979a). Contextual control of the extinction of conditioned fear. Learning \& Motivation, 10, 445-466.

Bouton, M. E., \& Bolles, R. C. (1979b). Role of conditioned contextual stimuli in reinstatement of extinguished fear. Journal of Experimental Psychology: Animal Behavior Processes, 5, 368-378.

Bouton, M. E., \& PECK, C. A. (1989). Context effects on conditioning, extinction, and reinstatement in an appetitive conditioning preparation. Animal Learning \& Behavior, 17, 188-198.

Bouton, M. E., \& RickER, S. T. (1994). Renewal of extinguished responding in a second context. Animal Learning \& Behavior, 22, 317-324.

Bouton, M. E., Rosengard, C., Achenbach, G. G., Peck, C. A., \& BrooKs, D. C. (1993). Effects of contextual conditioning and unconditional stimulus presentation on performance in appetitive conditioning. Quarterly Journal of Experimental Psychology, 46B, 6395 .

Bouton, M. E., \& Swartzentruber, D. (1989). Slow reacquisition following extinction: Context, encoding, and retrieval mechanisms. Journal of Experimental Psychology: Animal Behavior Processes, $15,43-53$.

Brogden, W. J., Lipman, E. A., \& Culler, E. (1938). The role of incentive in conditioning and extinction. American Journal of Psychology, 51, 109-117,

Bullock, D. H., \& Smith, W. C. (1953). An effect of repeated conditioning-extinction upon operant strength. Journal of Experimental Psychology, 46, 349-352.

CAPALDI, E. J. (1994). The sequential view: From rapidly fading stim- 
ulus traces to the organization of memory and the abstract concept of number. Psychonomic Bulletin \& Review, 1, 156-181.

DANGUIR, J., \& NicOLAIDIS, S. (1977). Lack of reacquisition in learned taste aversions. Animal Learning \& Behavior, 5, 395-397.

Delamater, A. R. (1995). Outcome-selective effects of intertrial reinforcement in a Pavlovian appetitive conditioning paradigm with rats. Animal Learning \& Behavior, 23, 31-39.

Farwell, B. J., \& AYres, J. J. B. (1979). Stimulus-reinforcer and response-reinforcer relations in the control of conditioned appetitive headpoking (goal tracking) in rats. Learning \& Motivation, 10 , 295-312.

FinCH, G., \& Culler, E. (1935). Relation of forgetting to experimental extinction. American Journal of Psychology, 47, 656-662.

FREY, P. W., \& BUTLER, C. S. (1977). Extinction after aversive conditioning: An associative or nonassociative process? Learning \& Motivation, 8, 1-17.

FrEY, P. W., \& Ross, L. E. (1968). Classical conditioning of the rabbit eyelid response as a function of interstimulus interval. Journal of Comparative \& Physiological Psychology, 65, 246-250.

Hall, G., \& Channell, S. (1985). Differential effects of contextual change on latent inhibition and on the habituation of an orienting response. Journal of Experimental Psychology: Animal Behavior Processes, 11, 470-481.

Hart, J. A., Bourne, M. J., \& Schachtman, T. R. (1995). Slow reacquisition of a conditioned taste aversion. Animal Learning \& Behavior, 23, 297-303.

Hilgard, E. R., \& Marquis, D. G. (1935). Acquisition, extinction, and retention of conditioned lid responses to light in dogs. Journal of Comparative Psychology, 19, 29-58.

HoEhLER, F. K., KIRSChenbaum, D. S., \& LeonaRd, D. W. (1973). The effects of overtraining and successive extinctions upon nictitating membrane conditioning in the rabbit. Learning \& Motivation, 4, 91-101.

Holland, P. C. (1979). Differential effects of omission contingencies on various components of Pavlovian appetitive conditioned responding in rats. Journal of Experimental Psychology: Animal Behavior Processes, 5, 178-193.

KaYe, H., \& Mackintosh, N. J. (1990). A change of context can enhance performance of an aversive but not of an appetitive conditioned response. Quarterly Journal of Experimental Psychology, 42B, 113-134.

KEHOE, E. J. (1988). A layered network model of associative learning: Learning to learn and configuration. Psychological Review, 95, 411-433.

Kehoe, E. J., \& Holt, P. E. (1984). Transfer across CS-US intervals and sensory modalities in classical conditioning of the rabbit. Animal Learning \& Behavior, 12, 122-128.

KeHoE, E. J., MoRrow, L. D., \& Holt, P. E. (1984). General transfer across sensory modalities survives reductions in the original conditioned reflex in the rabbit. Animal Learning \& Behavior, 12, 129136.

KonORSKI, J., \& SzWejkowsKa, G. (1950). Chronic extinction and restoration of conditioned reflexes: I. Extinction against the excitatory background. Acta Biologiae Experimentalis, 15, 156-170.
KonORSKI, J., \& SzWEJKowsKa, G. (1952a). Chronic extinction and restoration of conditioned reflexes: III. Defensive motor reflexes. Acta Biologiae Experimentalis, 16, 91-94.

KONORSKI, J., \& SZWEJKOWSKA, G. (1952b). Chronic extinction and restoration of conditioned reflexes: IV. The dependence of the course of extinction and restoration of conditioned reflexes on the "history" of the conditioned stimulus (the principle of the primacy of first training). Acta Biologiae Experimentalis, 16, 95-113.

Mackintosh, N. J. (1983). Conditioning and associative learning. Oxford: Oxford University Press.

MCAllister, D. E., \& MCAllister, W. R. (1994). Extinction and reconditioning of classically conditioned fear before and after instrumental learning: Effects of depth of fear extinction. Learning \& Motivation, 25, 339-367.

Napier, R. M., MaCrae, M., \& Kehoe, E. J. (1992). Rapid reacquisition in conditioning of the rabbit's nictitating membrane response. Journal of Experimental Psychology: Animal Behavior Processes, 18, 182-192.

Pearce, J. M., \& Hall, G. (1980), A model for Pavlovian learning: Variations in the effectiveness of conditioned but not of unconditioned stimuli. Psychological Review, 87, 532-552.

Pearce, J. M., \& Redhead, E. S. (1995). Supernormal conditioning. Journal of Experimental Psychology: Animal Behavior Processes, 21, 155-165.

Randich, A. (1981). The US preexposure phenomenon in the conditioned suppression paradigm: A role for conditioned situational stimuli. Learning \& Motivation, 12, 321-341.

Rescorla, R. A. (1987). A Pavlovian analysis of goal-directed behavior. American Psychologist, 42, 119-129.

RESCORLA, R. A., \& HETH, C. D. (1975). Reinstatement of fear to an extinguished conditioned stimulus. Journal of Experimental Psychology: Animal Behavior Processes, 1, 88-96.

RevuSKY, S., \& CoOMBEs, S. (1979). Reacquisition of learned taste aversions. Animal Learning \& Behavior, 7, 377-382.

Schreurs, B. G., \& Kehoe, E. J. (1987). Cross-modal transfer as a function of initial training level in classical conditioning with the rabbit. Animal Learning \& Behavior, 15, 47-54.

SMith, M., \& Gormezano, I. (1965). Effects of alternating classical conditioning and extinction sessions on the conditioned nictitating membrane response of the rabbit. Psychonomic Science, 3, 91-92.

SzWejKowsKa, G. (1950). The chronic extinction and restoration of conditioned reflexes: II. The extinction against an inhibitory background. Acta Biologiae Experimentalis, 15, 171-184.

WAGNER, A. R. (1969). Frustrative nonreward: A variety of punishment. In B. A. Campbell \& R. M. Church (Eds.), Punishment and aversive behavior (pp. 157-181). New York: Appleton-CenturyCrofts

WAGNER, A. R. (1981). SOP: A model of automatic memory processing in animal behavior. In N. E. Spear \& R. R. Miller (Eds.), Information processing in animals: Memory mechanisms (pp. 5-47). Hillsdale, NJ: Erlbaum.

(Manuscript received June 22, 1995; revision accepted for publication December 12, 1995.) 